\title{
Adaptive behaviour in children and adolescents with foetal alcohol spectrum disorders: a comparison with specific learning disability and typical development
}

\author{
Åse Fagerlund, \\ Folkhälsan Research Center, Paasikivigatan 4, 00250 Helsinki, Finland \\ ase.fagerlund@folkhalsan.fi \\ Department of Psychology and Logopedics, Åbo Akademi University, Biskopsgatan 3, 20500 \\ Turku, Finland

\section{Ilona Autti-Rämö,} \\ Hospital for Children and Adolescents, Department of Child Neurology, University of Helsinki, \\ Lastenlinnantie 2, 00250 Helsinki, Finland \\ Research Department, The Social Insurance Institute, Nordenskiöldinkatu 12, 00250 Helsinki, \\ Finland \\ Mirjam Kalland, \\ Folkhälsan Research Center, Paasikivigatan 4, 00250 Helsinki, Finland \\ Mannerheim League for Child Welfare, Toinen Linja 17, P.O. Box 141, 00531 Helsinki, Finland \\ Pekka Santtila, \\ Department of Psychology and Logopedics,Åbo Akademi University, Biskopsgatan 3, 20500 \\ Turku, Finland
}

\section{H. Eugene Hoyme,}

Department of Pediatrics, Sanford School of Medicine of The University of South Dakota and Sanford Children's Hospital, 1305 W. 18th St., P.O. Box 5039, Sioux Falls, SD 57117-5039, USA

Sarah N. Mattson, and

Center for Behavioural Teratology, San Diego State University, 6330 Alvarado Court, Suite 100, San Diego, CA 92120, USA

\section{Marit Korkman}

Institute of Behavioural Sciences, University of Helsinki, Helsinki, Finland

\section{Abstract}

Foetal alcohol spectrum disorders (FASD) is a leading cause of intellectual disability in the western world. Children and adolescents with FASD are often exposed to a double burden in life, as their neurological sequelae are accompanied by adverse living surroundings exposing them to further environmental risk. In the present study, the adaptive abilities of a group of children and adolescents with FASD were examined using the Vineland Adaptive Behaviour Scales (VABS) and compared to those of a group of IQ-matched children with specific learning disorder (SLD) as well as with typically developing controls (CON). The results showed significantly different 
adaptive abilities among the groups: Children with FASD performed worse than IQ-matched children with SLD, who in turn performed worse than typically developing children on all domains (communication, daily living skills and socialization) on the VABS. Compared to the other groups, social skills declined with age in the FASD group. These results support previous studies of adaptive behaviour deficits in children with FASD and provide further evidence of the specificity of these deficits. On a societal level, more efforts and resources should be focused on recognizing and diagnosing FASD and supporting communication skills, daily living skills and most of all social skills across diagnostic groups within FASD. Without adequate intervention, adolescents and young adults with FASD run a great risk of marginalization and social maladjustment, costly not only to society but also to the lives of the many young people with FASD.

\section{Keywords}

Foetal alcohol spectrum disorders; Foetal alcohol syndrome; Specific learning disability; Adaptive behaviour; Adaptive skills

\section{Introduction}

Disorders caused by prenatal alcohol exposure can be classified under different diagnostic categories that are drawn together under the umbrella term foetal alcohol spectrum disorders (FASD). Several published sets of diagnostic criteria for FASD are currently being used to identify and describe individuals exposed to alcohol prenatally, including the Revised Institute of Medicine (IOM) Diagnostic Classification System [1] and the 4-Digit Diagnostic Code $[2,3]$. All guidelines rely on anomalies in three distinct areas: prenatal and/or postnatal growth deficiency, central nervous system dysfunction, and a pattern of facial characteristics. In the following, the Revised IOM Diagnostic Classification System will be described (for detailed comparisons between diagnostic schemata please see Warren et al. [4] and Riley et al. [5]). Foetal alcohol syndrome (FAS) is characterized by typical facial features (short palpebral fissures, thin vermilion border of the upper lip and smooth philtrum), growth retardation and deficient brain growth. Affected individuals not fulfilling the FAS diagnostic criteria can receive a diagnosis of partial FAS (PFAS), alcohol-related neurodevelopmental disorder (ARND) or alcohol-related birth defects (ARBD). For a PFAS diagnosis, the facial characteristics have to be present as well as growth retardation, deficient brain growth or evidence of a complex pattern of behavioural and cognitive abnormalities inconsistent with developmental level. Importantly, individuals with ARND do not display the typical facial features of FAS, but demonstrate either evidence of deficient brain growth or a complex pattern of behaviour or cognitive abnormalities inconsistent with the developmental level that can be linked to documented prenatal alcohol exposure.

Individuals with FASD often face a double burden in life. Not only have they been exposed to a hazardous teratogen during prenatal development, jeopardizing brain development, but they are also often born into families where at least one parent continues to abuse alcohol. Many of the children experience non-optimal rearing environments and may at some stage be separated from their parents and placed in foster homes or residential care [6]. In absolute numbers, the FASD group is substantial: prenatal alcohol exposure is considered a leading cause of intellectual disability in the western world, more common than, for example, Down's syndrome or Cerebral Palsy [7, 8]. Two prevalence studies conducted in Europe have found overall prevalence rates for FASD as high as $2.3-4.1 \%$ in Italy [9] and $4.1 \%$ in Croatia [10]. In the US, the prevalence of FASD is similarly estimated to range between 2 and 5\% [11]. However, compared to prevalences found using in-school studies, only 1 out of 
7-10 children (10-14\%) receive their FAS diagnosis through the general health care system; and in the case of children with PFAS or ARND diagnoses are made even less often [12]. Thus, within the population of individuals with FASD there exist a large group of undiagnosed children who are subject to structural and functional disabilities due to both prenatal damage from a teratogen and the long-term consequences of a social disadvantage. In addition, there may be detrimental effects of maternal prenatal stress affecting neurodevelopment $[13,14]$. An important question is how well children and adolescents growing up with FASD will adapt to the world around them, develop skills to cope with everyday demands and learn to live independently.

Adaptive behaviour can be defined as the performance of daily activities required for personal and social self-sufficiency or the ability to respond successfully to everyday demands [15]. In other words, adaptive abilities are about developing skills that gradually enable an independent life, maintain social relationships, and allow the individual to become integrated into society. Adaptive skills are closely age related and defined by performance rather than ability. According to the Vineland Adaptive Behaviour Scales (VABS), adaptive behaviour can be conceptualized in three domains: communication (receptive, expressive and written), daily living skills (how an individual eats, dresses, performs household tasks, uses time, money, the telephone and performs at work), and socialization (how an individual interacts with others, plays and uses leisure time, as well as the development of coping skills, i.e., how an individual demonstrates responsibility and sensitivity to others) [15].

Deficits in adaptive behaviour are observed in different groups of children with developmental difficulties, e.g., autism [16], intellectual disability [17] and ADHD [18]. The relatively few studies performed on adaptive abilities in children with prenatal exposure to alcohol show somewhat mixed results. According to Coles et al. [19], prenatal alcohol exposure in the absence of the typical somatic features and the mental retardation associated with full FAS does not impair social and adaptive abilities. In contrast, Carr et al. [20] argue that adaptive difficulties are found regardless of the diagnosis received under the FASD umbrella.

If we are to delineate a specific adaptive profile for children with FASD, their adaptive behaviour has to be contrasted with that of other groups of children. Jirikowic et al. [21] compared 5-8-year-olds with FASD to same aged typically developing children and found clear differences with regard to adaptive skills. Crocker et al. [18], comparing 6-13-yearolds with FASD and ADHD on adaptive behaviour, came to the conclusion that both groups were impaired on adaptive behaviour, but that children in the FASD group were significantly more impaired than the ADHD group on daily living skills. However, in these studies the children were not matched on IQ (even though Crocker et al. managed to match a small subgroup of their sample on IQ and found results pointing in the same direction). If IQ is not taken into account the possibility that differences between groups are a consequence of a lower IQ in the FASD group cannot be excluded. Accordingly, Whaley et al. [22] found no significant differences between 2 to 10 -year-old alcohol-exposed children versus a very heterogeneous group of non-exposed clinical control children when the groups were matched on IQ.

The studies also vary as to which adaptive domain is most affected in alcohol-exposed children. While one study showed the weakest scores on daily living skills and best scores on social skills [18] the opposite was true in another [23]. There is more agreement on the fact that impairments in social skills become more marked with age in the FASD group [18, $22,24]$ or at least that difficulties with social skills are apparent in both younger and older age groups [23, 25]. 
As children with FASD not only bear the burden of neurological sequelae, but also may experience an adverse rearing environment, the reasons behind difficulties with adaptive behaviour could hypothetically be twofold: stemming from neurocognitive difficulties, a non-optimal environment or, more likely, a combination of both. Extensive research has shown that prenatal alcohol exposure can result in vast neurocognitive difficulties [26, 27] including deficient executive functions [28, 29]. An individual experiencing difficulties with executive functions may have problems modifying behaviour in response to changes in the environment, solving problems, or focusing and shifting attention. It is not unexpected that this individual would also experience challenges in everyday adaptive functioning. Thus, executive function deficits in FASD could be seen as a link to impaired adaptive behaviour.

Rearing environment and out of home placement may also play a factor in the adaptive abilities of children with FASD. A Finnish study showed that $50 \%$ of children born to mothers with alcohol and drug abuse were taken into custody over a 2-12-year follow-up and placed either in foster- or adoptive families or within residential care [6]. FASD children growing up in residential care units separated from their parents have been shown to be most at risk for manifesting severe behavioural problems compared to FASD children living with their biological parents or in foster homes [30]. Other studies have shown insecure attachment rates as high as 70-80\% in children with FASD [31]. In support of the notion that rearing environment may influence the adaptive abilities of individuals with FASD, maltreated children with a diagnosis of reactive attachment disorder showed developmental delays in all adaptive domains [32]. To our knowledge, however, no study has taken rearing environment into account when reporting on adaptive skills in the FASD group.

In summary, no clear adaptive profile-distinguishing individuals with FASD from other groups with developmental diagnoses has been demonstrated, even though social impairments seem to be a characteristic of this group. Samples are often small and there is a lack both of comparisons to other developmental disorders as well as incomplete matching of groups on important variables, such as IQ and/or caregiving environment. For children and adolescents with FASD, both neurocognitive difficulties as well as an adverse rearing could result in developmental gaps compared to other children impairing adaptive functioning in life.

The purpose of the present study was to compare adaptive behaviour in children and adolescents with histories of prenatal exposure to alcohol (FASD) with two contrast groups: an IQ-matched group of children with specific learning disability (SLD) and a control group of typically developing children (CON). A smaller subgroup of children was matched on caregiving environment. Based on earlier studies, it was predicted that: (a) in comparison with children with SLD and typically developing children, the FASD group would show more impaired adaptive behaviour than could be expected on the basis of IQ alone; (b) the group differences, especially for social skills, among the FASD group and the two comparison groups would increase with age; (c) the developmental gap between SLD children and children with FASD would diminish if continuity of caregiving environment is taken into account.

\section{Methods}

\section{Participants}

A total of 143 children and adolescents were included in this study. The participants consisted of three groups: one group with histories of prenatal exposure to alcohol, the FASD group $(N=73)$; one IQ-matched contrast group consisting of children with mostly specific learning disorder (SLD, $N=30$ ); and a typically developing control group (CON, $N$ $=40)$. 
FASD group-The FASD group comprised 44 females and 29 males diagnosed with FAS $(N=41,56.2 \%)$, PFAS $(N=23,31.5 \%)$ and ARND $(N=9,12.3 \%)$ (see Table 1$)$. Participants were recruited through review of medical records from three hospitals in the Helsinki area (Helsinki University Hospital, Children's Castle Hospital and Aurora District Hospital). Patients with a diagnosis of FAS or FAE (an older non-specific term referring to children adversely affected by prenatal alcohol exposure who display some, but not all, of the features of FAS) as well as those with a confirmed history of heavy prenatal alcohol exposure during pregnancy were selected. All selected participants were then examined by an experienced dysmorphologist (HEH) who evaluated and confirmed diagnoses in the foetal alcohol spectrum using modified Institute of Medicine Criteria [1,33]. Any cases with patterns of malformation inconsistent with an FASD were referred for further clinical genetics evaluation and/or testing. For further information regarding screening and evaluation procedures, see Autti-Rämö et al. [12].

SLD group-In order to find neurodevelopmental and adaptive characteristics specific to FASD, we included a contrast group of children with specific learning difficulties and comparable cognitive level. In clinical practice, those with SLD come to the same neurological examination units as FASD patients and are evaluated for similar rehabilitation procedures and special education. To allow for IQ matching, 8 children had an IQ below 70 and the rest $(N=22)$ an IQ above 70 . This group had been diagnosed with either mixed specific developmental disorder (coded F83 according to the ICD-10 [34]) or specific developmental disorder of scholastic skills (F81 according to the ICD-10 [34]). Mixed specific developmental disorder is described as a category in which there is a mixture of specific developmental disorders of speech and language, of scholastic skills, and of motor function, but in which none predominates sufficiently to constitute the prime diagnosis. The disorder is usually, but not always, associated with some degree of general impairment of cognitive functions [34]. F81, Specific developmental disorder of scholastic skills, is a disorder where the normal pattern of skill acquisition regarding reading, spelling, writing, and arithmetical skills is disturbed from the early stages of development. This should not simply be a consequence of a lack of opportunity to learn, not solely a result of mental retardation, and not due to any form of acquired brain trauma or disease [34]. The 30 children and adolescents included in the SLD group were matched with the FASD group on IQ, age and sex. They were recruited from the same three hospitals in the Helsinki area as the FASD group. For patients diagnosed with F83 or F81 every effort to exclude FASD in the SLD group was done by strict inclusion criteria: for the SLD group (1) no history of maternal alcohol abuse in any of the medical journals, (2) no clinical signs including facial features suggestive of prenatal alcohol exposure in any of the medical records, (3) normal somatic growth according to growth charts, (4) maternal confirmation of no alcohol consumption during pregnancy. Overall, 59 possible participants were contacted; 30 agreed to participate and 29 did not. All participants who agreed to participate underwent clinical examination by two experienced dysmorphologists (HEH and Luther K. Robinson). Parents/ guardians were questioned about alcohol intake during pregnancy to eliminate the risk that any participant in the SLD group could have been exposed to alcohol during pregnancy.

CON group—Finally, a typically developing control group $(N=40)$ was recruited through random sampling from the Finnish national population registry, matched to the FASD subjects on age, sex and geographical region. 72 potential participants were contacted and 40 agreed to participate. As in the SLD group, all participants were examined by two dysmorphologists (HEH and Luther K. Robinson). Parents were questioned about alcohol intake during pregnancy and any mention of alcohol intake during pregnancy was an exclusion criterion in the CON group. 


\section{Procedures}

All selected participants or their parents/foster parents/legal guardians were initially contacted by phone. Those interested in participating received a letter with further information regarding the study. After a few days, a research assistant contacted them again to schedule an appointment at the Folkhälsan Research Center in Helsinki. At the clinic, all participants (caregivers as well as the children) read and signed forms of informed consent or assent agreements prior to examinations. Caregivers were interviewed in person by a nurse experienced in working with FASD at which time an extensive family history was taken and the Vineland Adaptive Behaviour Scales (VABS) was conducted. As part of a larger test battery, intelligence testing was carried out with each child/adolescent by a clinical psychologist while the caregiver was interviewed in a separate room. By the end of testing, children and adolescents were given 20-Euro compensation in the form of a gift card. The screening and research procedures were approved by the Coordinating Ethics Committee of the Helsinki and Uusimaa Hospital District and the San Diego State University Institutional Review Board.

\section{Measures}

Adaptive behaviour-The VABS was developed as a standardized measure to assess an individual's level of adaptive daily functioning from birth to 18 years 11 months old or a low functioning adult [15]. In this study, the VABS Interview Edition-Survey Form was used, administered in the form of a semi-structured interview with a parent or caregiver. The VABS assesses adaptive behaviour in three domains, each of which is divided into three subdomains: communication (receptive, expressive and written), daily living skills (personal, domestic, and community) and socialization (interpersonal relationship skills, play and leisure time, and coping skills). In addition, an adaptive behaviour composite score can be derived from the three domains.

Intelligence-Wechsler Intelligence Scale for Children-III (WISC-III) and Wechsler Adult Intelligence Scale-III (WAIS-III) are well-known standardized tests for measuring cognitive abilities [35]. The WISC-III is intended for use with children aged 6-15 years and the WAIS-III for 16 year olds and above. In this study, a shortened version of the Finnish standardized versions of the tests was used. The subtests included three verbal tests and three performance tests: Information, Vocabulary and Comprehension for verbal IQ and Picture Completion, Picture Arrangement, and Block Design for performance IQ.

In addition, the Brief IQ screener from the Leiter International Performance Scale-Revised (LIPS-R) was included to verify the results on the Wechsler scales. LIPS-R is a measure of nonverbal intelligence in fluid reasoning and visualization developed for children and adolescents from 2 to 20 (Roid \& Miller ref). The brief IQ screener consists of four subtests for estimation of global intellectual ability.

Statistical analyses-All data were entered, and statistical calculations performed, using IBM SPSS Statistics version 19 [36]. Descriptive statistics were calculated for the three groups separately. Matching criteria was controlled using one-way analysis-of-variance (age, IQ) and the chi-square test (sex). In the subgroup analysis of the FASD and SLD children matching criteria was controlled using independent sample $t$ tests (age, IQ) and the chi-square test (sex). Pearson correlations were performed to correlate IQ and adaptive behaviour. One-way analyses of variance were used to compare the three groups on adaptive behaviour and to compare adaptive skills within the FASD group. A subgroup analysis between the FASD and SLD children raised in biological families was performed with the nonpara-metric Mann-Whitney $U$ test. 


\section{Results}

\section{Demographic characteristics}

The three groups were similar with respect to age $\left[F_{(2,140)}=0.217, P=.805\right]$ and $\operatorname{sex}\left[\chi^{2}(d f\right.$ $=2)=0.543, P=.762]$, but differed on IQ $\left[F_{(2,138)}=41.813, P=.000\right]$. Pairwise comparisons employing the Duncan post-hoc test indicated that there was no significant difference between the FASD group and the SLD group $(P=.340)$. However, the FASD group and the SLD group were both significantly different from the CON group $(P=.000)$. Demographic information is detailed in Table 1.

\section{Adaptive behaviour and IQ}

Correlations were run between IQ and adaptive behaviour scores for all 143 participants. Significant correlations were found for adaptive behaviour composite score $(r=0.566, P=$. $000)$ and for the domain scores for communication $(r=0.503, P=.000)$, daily living skills $(r$ $=0.573, P=.000)$, and socialization $(r=0.418, P=.000)$. On a group level, significant correlation between IQ and adaptive behaviour scores was found for the FASD group on adaptive behaviour composite score $(r=0.410, P=.000)$, communication domain $(r=$ $0.375, P=.001)$ and daily living skills $(r=0.503, P=.000)$. In the CON group daily living skills and IQ correlated moderately $(r=0.356, P=.024)$. No other correlations on group level were found across the groups.

\section{Comparison of groups on adaptive behaviour domains and subdomains}

Separate analyses of variance were conducted to evaluate the effect of group (FASD, SLD, CON) on the VABS composite score, domains (3) and subdomains (9). As can be seen in Table 2, significant differences among the groups were revealed for all scores except the subdomain receptive communication. On the composite score and domain levels, a clear pattern was revealed: the typically developing controls scored better than the children with learning disorders, who in turn scored better than the alcohol-exposed children. On a subdomain level, the FASD group still scored worse than both other groups on most scales (see Table 2).

\section{Combined influences of group and age on adaptive behaviour}

To address the broader question of whether age influenced adaptive behaviour skills, we divided the sample into two age groups as equally as possible; 8-12 year olds $(N=73)$ and 13-20 year olds $(N=70)$. A two-way analysis of variance (General Linear Model) was performed combining age and group. Results revealed a significant two-way interaction for the socialization domain (Table 3 and Fig. 1, note that the main effects of group correspond to data presented in Table 2). In the FASD group, socialization skills were worse in the older age group (13-20 year olds) compared to the younger children (8-12 year olds). In the SLD group, on the other hand, socialization skills improved with age and approached the typically developing control children.

\section{Comparison of caregiving environment between the FASD and SLD groups}

As the FASD group consistently performed less well on adaptive behaviour skills compared not only to normal controls but also to their IQ-matched peers in the SLD group, we wanted to explore whether caregiving environment affected this relationship. Most children in the FASD group had at some stage been taken into custody and thus experienced a separation from their biological family. At the time of the study, 75.3\% $(N=55)$ of them lived in foster- or adoptive homes with a continuous caregiver, $16.4 \%(N=12)$ lived in residential care with alternating caregivers and $8.2 \%(N=6)$ had been able to stay in their biological 
families. Differences between these groups on adaptive behaviour skills did not reach significant levels.

We then compared the subgroup of children with FASD living in their biological families $(N$ $=6)$ with a corresponding group of SLD children living in their biological families $(N=28$, excluded in the analysis were one child in the SLD group who lived with foster parents and one who was in residential care). Of the six children with FASD, two had a diagnosis of FAS, two had a diagnosis of PFAS and two a diagnosis of ARND. The groups were still similar with respect to sex $\left[\chi^{2}(d f=1)=0.551, P=.458\right]$, age $[t(32)=0.342, P=.734]$ and IQ [t(32) $=-0.738, P=.466]$ but now also on caregiving environment (being raised in one's biological family). As the number of participants in the FASD group was very small, the analysis was performed using the nonparametric independent samples Mann-Whitney $U$ test. Even though absolute numbers indicate lower scores in the FASD group compared to the SLD group (see Table 4), results no longer showed any significant differences between the two groups on the VABS composite score, domain or subdomain scores.

\section{Adaptive behaviour skills in the FASD group}

Finally, to examine further the adaptive skills within the FASD group separate analyses were performed for diagnostic subgroups. Significant differences were found on the adaptive behaviour composite score $\left(F_{(2,70)}=3.43, P=.038\right.$, FAS and PFAS < ARND) as well as on the domain scores of communication $\left(F_{(2,71)}=4.12, P=.020\right.$, FAS and PFAS < ARND) and daily living skills $\left(F_{(2,71)}=3.92, P<.024\right.$, FAS $<$ ARND). Diagnostic category was not associated with socialization scores.

\section{Discussion}

In the present study, clearly different adaptive profiles among children and adolescents with FASD, SLD and typically developing controls $(\mathrm{CON})$ were revealed. The FASD group performed worse than the SLD group, who in turn performed worse than the CON group on the adaptive behaviour composite, all domains (communication, daily living skills and socialization) and most subdomains on the VABS. Importantly, the SLD group outperformed the FASD group on adaptive behaviour in spite of comparable cognitive levels. This is the first study to compare adaptive abilities in a group of children and adolescents with FASD relative to both a contrast group of IQ-matched children with SLD and to a group of typically developing peers. In addition, a subgroup of children was matched on caregiving environment.

The present study supports the view that typically developing children outperform children with FASD on adaptive skills [21], and extends the understanding of adaptive abilities in FASD compared to IQ-matched children and adolescents with SLD. Earlier work on adaptive abilities in FASD has shown mixed results both within the FASD group [19, 20] and, in a limited fashion, in comparison to other clinical groups [18, 22]. In one study, where children with FASD were compared to a diverse group of IQ-matched clinical controls results showed no differences on adaptive abilities between groups [22]. In contrast, the clearly differential adaptive profiles of children with FASDs compared to children with SLD in this study may result from the combination of the well-defined IQ-matched comparison group and the inclusion of older participants in this study. It has been suggested that difficulties with adaptive abilities in FASD may become more prominent with age [22, 24].

In clinical practice, SLD is an important comparison group to FASD as these children often come to the same developmental neurology units where both groups may present with similar difficulties with learning. As diagnoses within the FASD continuum are difficult to make, children with FASD remain remarkably underdiagnosed [11, 37]. However, as 
children with FASD and SLD show differential patterns of adaptive skills regardless of IQ, the importance of accurately and differentially diagnosing FASDs is underscored.

Further analyses combining effects of age and group showed declining socialization skills with age in the FASD group. In contrast to this, children in the SLD group improved their socialization skills with age; as adolescents, their level of social skills approached that of the CON group. The results thus support the theory that deficits in social skills may increase with age among individuals with FASD [18, 24]. Typically, during late childhood and adolescence, there are important changes in social awareness associated with a comparatively late development of brain regions regulating social processes. The theories on the development of social decision-making suggest an interaction between an innate, emotion-inducing system and an acquired, emotion-regulating system gradually developing over time. Possible regulatory mechanisms pertain to the development of cognitive control (the ability to keep relevant information in an active state and exert goal-directed behaviour), future orientation (the ability to anticipate future consequences of actions) and perspective taking (consider the thoughts and perspectives of other people) which are thought to be regulated by different neural networks in the brain and gradually mature during late childhood and adolescence [38]. According to these theories, social deficits in FASD might be associated with either a later than normal maturation of one or more of these regulating systems, (partly) deviantly functioning systems, or a combination of both.

Research has shown that adolescents and adults with FASD often face a dependent living situation and have problems with employment $[25,39]$. Young adults with FASD also experience serious problems with their own parenthood (40\% of FASD females drank during pregnancy) $[25,39,40]$. The adult lives of many are characterized by alcohol and drug problems, trouble with the law and disrupted school experiences [40]. While on a societal level the estimated lifetime cost per person with FASD is about 1.3 million euro [41], the effect on an affected individual in terms of human cost may be immeasurable. In clinical practice, every effort has to be made to intervene and find ways to improve adaptive abilities of all children growing up with FASD.

Caregiving environment also seemed tied to level of adaptive skills, although results are very tentative as the analysis was performed on a small subgroup and the statistical power is low. When children with FASD and SLD living in their birth families were compared, the developmental gap between groups diminished considerably, supporting the notion that adaptive impairments in individuals with FASD cannot be explained by neurological sequelae alone but are also influenced by the rearing environment. In the FASD group the children not living with their biological parents had all been taken into custody and subsequently been placed in foster/adoptive homes with continuous caregivers or in residential care with turnover of caregivers. In Finland, reasons behind child welfare actions pertain to circumstances endangering the child's development [42]. In other words, prior to intervention by child welfare, the children had experienced adverse family environments such as alcohol abuse and violence [43].

One possible solution to the diminished developmental gap between children in biological families compared to children in care may be found in attachment theory. According to this theory, human infants develop a hierarchy of a small number of preferred attachment figures during the first year of life theoretically developing as a mechanism to assure the survival of the child $[44,45]$. Research on institutionalized children has shown that the development of normal social behaviour is not dependent on the physical quality of the environment, but upon the presence of a loving and stable caregiver [44] and that securely attached children exhibit better social skills and a more developed theory of mind than less securely attached children [46]. Accordingly, children who have been moved into foster care often show 
insecure attachment patterns 1 year after placement [47]. If a birth mother of a child born with FASD receives support enough to take care of her child and form a reasonably secure attachment bond, the child may be saved from the emotionally painful separation connected to being taken into custody. As an adverse environment has been linked to level of adaptive abilities [32], it might be speculated that the better adaptive abilities in FASD children living in their (recovered) birth families could reflect a relatively stable and secure environment. If this is true, more efforts should be made to support birth mothers in their recovery and support birth families to take care of their alcohol-affected children when possible.

In contrast to earlier work [22], adaptive abilities were moderately correlated to IQ across comparison groups in this study. Interestingly, on a group level, IQ was related to adaptive abilities in the FASD group on all domains but the social skills domain. Within the FASD group socialization was also the only domain where level of adaptive ability was not tied to diagnostic category. In addition, younger participants ( 8 to 12 -year olds) performed better on social skills compared to older participants (17 to 20-year olds). The results lend support to the notion from studies with younger children that social abilities seem to be especially affected in FASD $[18,24]$ and extend results to adolescents with FASD regardless of overall ability level.

The present study has several strengths. It presents a comparatively large group of carefully diagnosed children and adolescents across the continuum of FASD compared to an IQ, age and sex-matched contrast group of children with SLD as well as to a group of typically developing peers. In addition, we were able to match a smaller subsample on caregiving environment. As this sample also includes teenagers, a wider age range can be addressed than in most previous studies on FASD and adaptive skills. A limitation of this study is the small size of the FASD subgroup compared with SLD on caregiving environment. Ideally, to delineate an adaptive profile for FASD both IQ and caregiving environment should be fully accounted for. However, in practice this is difficult to achieve.

\section{Conclusion}

In conclusion, children and adolescents with FASD are often exposed to a double burden in life, as their neurological sequelae are accompanied by adverse living surroundings exposing them to further environmental risk. In addition, they remain remarkably underdiagnosed by the health care system. The results of this study show that the adaptive abilities of children and adolescents growing up with FASD are significantly compromised compared to both typically developing peers and IQ-matched children with SLD. On a societal level, more efforts and resources should be focused on recognizing and diagnosing FASD and supporting communication skills, daily living skills and most of all social skills across diagnostic groups within FASD. Without adequate intervention, adolescents and young adults with FASD run a great risk of marginalization and social maladjustment, costly not only to society but also to the lives of the many young people with FASD.

\section{Acknowledgments}

The authors thank all the children, adolescents and adults who invested their time and effort to participate in this study. We also gratefully acknowledge our co-workers Leena Loimu, Leena Neuvonen, Kirsi Mallea, Nina Ervalahti and Luther Robinson for valuable input. The data collection phase of this study was supported by Grant U01 AA014834 from the National Institute on Alcohol Abuse and Alcoholism (NIAAA) in the United States. Analysis and reporting was supported by the Folkhälsan Research Center,Åbo Akademi University and the Victoria foundation in Finland. 


\section{References}

1. Hoyme HE, May PA, Kalberg WO, Kodituwakku P, Gossage JP, Trujillo PM, Buckley DG, Miller JH, Aragon AS, Khaole N, Viljoen DL, Jones KL, Robinson LK. A practical clinical approach to diagnosis of fetal alcohol spectrum disorders: clarification of the 1996 institute of medicine criteria. Pediatrics. 2005; 115:39-47. [PubMed: 15629980]

2. Astley SJ. Comparison of the 4-digit diagnostic code and the Hoyme diagnostic guidelines for fetal alcohol spectrum disorders. Pediatrics. 2006; 118(4):1532-1545. [PubMed: 17015544]

3. Astley, SJ. Diagnostic guide for Fetal Alcohol Spectrum Disorders. The 4-Digit Diagnostic Code. 3rd edn.. University of Washington; Seattle: 2004.

4. Warren KR, Hewitt BG, Thomas JD. Fetal alcohol spectrum disorders. Research challenges and opportunities. Alcohol Res Health J Natl Inst Alcohol Abuse Alcohol. 2011; 34(1):4-14.

5. Riley EP, Infante MA, Warren KR. Fetal alcohol spectrum disorders: an overview. Neuropsychol Rev. 2011; 21(2):73-80. [PubMed: 21499711]

6. Sarkola T, Kahila H, Halmesmäki E. Risk factors for out-of-home custody child care among families with alcohol and substance abuse problems. Acta Pediatrica. 2007; 96:1571-1576.

7. Marttala J, Yliniemi O, Gissler M, Nieminen P, Ryynanen M. Prevalence of Down's syndrome in a pregnant population in Finland. Acta Obstet Gynecol Scand. 2010; 89(5):715-717. [PubMed: 20218932]

8. Arneson CL, Durkin MS, Benedict RE, Kirby RS, Yeargin-Allsopp M, Van Naarden Braun K, Doernberg NS. Prevalence of cerebral palsy: Prevalence of cerebral palsy: autism and developmental disabilities monitoring network, three sites, United States, 2004. Disabil Health J. 2009; 2(1):45-48. [PubMed: 21122742]

9. May PA, Fiorentino D, Phillip Gossage J, Kalberg WO, Eugene Hoyme H, Robinson LK, Coriale G, Jones KL, del Campo M, Tarani L, Romeo M, Kodituwakku PW, Deiana L, Buckley D, Ceccanti M. Epidemiology of FASD in a province in Italy: prevalence and characteristics of children in a random sample of schools. Alcohol Clin Exp Res. 2006; 30(9):1562-1575. [PubMed: 16930219]

10. Petkovic G, Barisic I. FAS prevalence in a sample of urban schoolchildren in Croatia. Reprod Toxicol. 2010; 29(2):237-241. [PubMed: 19922788]

11. May PA, Gossage JP, Kalberg WO, Robinson LK, Buckley D, Manning M, Hoyme HE. Prevalence and epidemiologic characteristics of FASD from various research methods with an emphasis on recent in-school studies. Dev Disabil Res Rev. 2009; 15(3):176-192. [PubMed: 19731384]

12. Autti-Rämö I, Ritvanen A. Miten selvitämme, lisääntyvätkö alkoholin aiheuttamat sikiövauriot? Suomen Lääärilehti. 2005; 60(12-13):1388-1389.

13. Mathews HL, Janusek LW. Epigenetics and psychoneuroimmunology: mechanisms and models. Brain Behav Immun. 2011; 25(1):25-39. [PubMed: 20832468]

14. Talge NM, Neal C, Glover V. Early Stress, Translational Research and Prevention Science Network: fetal and neonatal experience on child and adolescent mental health: antenatal maternal stress and long-term effects on child neurodevelopment: how and why? J Child Psychol Psychiatry. 2007; 48(3-4):245-261. [PubMed: 17355398]

15. Sparrow, SS.; BAlla, DA.; Cicchetti, DV. Vineland Adaptive Behavior Scales. interview edition. American Guidance Services, Inc.; Circle Pines: 1984.

16. Liss M, Harel B, Fein D, Allen D, Dunn M, Feinstein C, Morris R, Waterhouse L, Rapin I. Predictors and correlates of adaptive functioning in children with developmental disorders. $\mathrm{J}$ Autism Dev Disord. 2001; 31(2):219-230. [PubMed: 11450820]

17. Tremblay KN, Richer L, Lachance L, Cote A. Psychopathological manifestations of children with intellectual disabilities according to their cognitive and adaptive behavior profile. Res Dev Disabil. 2010; 31(1):57-69. [PubMed: 19700260]

18. Crocker N, Vaurio L, Riley EP, Mattson SN. Comparison of adaptive behavior in children with heavy prenatal alcohol exposure or attention-deficit/hyperactivity disorder. Alcohol Clin Exp Res. 2009; 33(11):2015-2023. [PubMed: 19719794] 
19. Coles CD, Brown RT, Smith IE, Platzman KA, Erickson S, Falek A. Effects of prenatal alcohol exposure at school age. I. Physical and cognitive development. Neurotoxicol Teratol. 1991; 13(4): 357-367. [PubMed: 1921915]

20. Carr JL, Agnihotri S, Keightley M. Sensory processing and adaptive behavior deficits of children across the fetal alcohol spectrum disorder continuum. Alcohol Clin Exp Res. 2010; 34(6):10221032. [PubMed: 20374212]

21. Jirikowic T, Kartin D, Olson HC. Children with fetal alcohol spectrum disorders: a descriptive profile of adaptive function. Can J Occup Ther. 2008; 75(4):238-248. [PubMed: 18975670]

22. Whaley SE, O'Connor MJ, Gunderson B. Comparison of the adaptive functioning of children prenatally exposed to alcohol to a nonexposed clinical sample. Alcohol Clin Exp Res. 2001; 25(7): 1018-1024. [PubMed: 11505027]

23. Streissguth AP, Aase JM, Clarren SK, Randels SP, LaDue RA, Smith DF. Fetal alcohol syndrome in adolescents and adults. JAMA. 1991; 265(15):1961-1967. [PubMed: 2008025]

24. Thomas SE, Kelly SJ, Mattson SN, Riley EP. Comparison of social abilities of children with fetal alcohol syndrome to those of children with similar IQ scores and normal controls. Alcohol Clin Exp Res. 1998; 22(2):528-533. [PubMed: 9581664]

25. Spohr H, Willms J, Steinhausen H. Fetal alcohol spectrum disorders in young adulthood. J Pediatr. 2007; 150:175-179. [PubMed: 17236896]

26. Kodituwakku PW. Defining the behavioral phenotype in children with fetal alcohol spectrum disorders: a review. Neurosci Biobehav Rev. 2007; 31(2):192-201. [PubMed: 16930704]

27. Mattson SN, Schoenfeld AM, Riley EP. Teratogenic effects of alcohol on brain and behavior. Alcohol Res Health. 2001; 25(3):185-191. [PubMed: 11810956]

28. Kodituwakku PW, Kalberg W, May PA. The effects of prenatal alcohol exposure on executive functioning. Alcohol Res Health. 2001; 25(3):192-198. [PubMed: 11810957]

29. Rasmussen C. Executive functioning and working memory in fetal alcohol spectrum disorder. Alcohol Clin Exp Res. 2005; 29(8):1359-1367. [PubMed: 16131842]

30. Fagerlund A, Autti-Ramo I, Hoyme HE, Mattson SN, Korkman M. Risk factors for behavioural problems in foetal alcohol spectrum disorders. Acta Paediatr. 2011; 100:1481-1488. [PubMed: 21575054]

31. O'Connor MJ, Kogan N, Findlay R. Prenatal alcohol exposure and attachment behavior in children. Alcohol Clin Exp Res. 2002; 26(10):1592-1602. [PubMed: 12394294]

32. Becker-Weidman A. Effects of early maltreatment on development: a descriptive study using the Vineland Adaptive Behavior Scales-II. Child Welfare. 2009; 88(2):137-161. [PubMed: 19777796]

33. Stratton, KR.; Howe, CJ.; Battaglia, FC. Fetal Alcohol Syndrome: diagnosis, epidemiology, prevention, and treatment. National Academy Press; Washington, DC: 1996.

34. World Health Organization. International statistical classification of diseases and related health problems - tenth revision. 2008 edn.. World Health Organization; Geneva: 2009.

35. Wechsler, D. Wechsler Intelligence Scale for children. 3rd edn.. The Psychological Corporation; San Antonio, TX: 1991.

36. IBM corporation. IBM SPSS statistics. 2011:19.

37. Vagnarelli F, Palmi I, Garcia-Algar O, Falcon M, Memo L, Tarani L, Spoletini R, Pacifici R, Mortali C, Pierantozzi A, Pichini S. A survey of Italian and Spanish neonatologists and paediatricians regarding awareness of the diagnosis of FAS and FASD and maternal ethanol use during pregnancy. BMC Pediatr. 2011; 11(1):51. [PubMed: 21645328]

38. Crone, EA.; Westenberg, PM. A brain-based account of developmental changes in social decision making.. In: De Haan, M.; Gunnar, RM., editors. Handbook of developmental social neuroscience. The Guilford Press; New York: 2009. p. 378-396.

39. Streissguth AP, Barr HM, Kogan J, Bookstein FL. Final report: understanding the occurrence of secondary disabilities in clients with fetal alcohol syndrome (FAS) and fetal alcohol effects (FAE). 1996:71.

40. Streissguth AP, Bookstein FL, Barr HM, Sampson PD, O'Malley K, Kogan J. Risk factors for adverse life outcomes in fetal alcohol syndrome and fetal alcohol effects. Dev Behav Pediatr. $2004 ; 25(4): 228-238$. 
41. Thanh, NX.; Jonsson, E.; Dennett, L.; Jacobs, P. Costs of FASD.. In: Riley, EP.; Clarren, S.; Weinberg, J.; Jonsson, E., editors. Fetal alcohol spectrum disorder. Management and policy perspectives of FASD. Wiley-Blackwell; Weinheim: 2011.

42. http://www.stakes.fi/EN/tilastot/statisticsbytopic/childhoodandfamily/childwelfare.htm

43. Koponen A. Katkennein siivin elämään. Sikiöaikana päihteille altistuneet lapset. 2005

44. Reeb, BC.; Fox, NA.; Nelson, CA.; Zeanah, CH. The effects of early institutionalization on social behavior and underlying neural correlates.. In: De Haan, M.; Gunnar, RM., editors. Handbook of developmental social neuroscience. The Guilford Press; New York: 2009. p. 477-496.

45. Zeanah, CH.; Boris, NW. Disturbances and disorders of attachment in early childhood.. In: Zeanah, $\mathrm{CH}$., editor. Handbook of infant mental health. 2nd edn.. The Guildford Press; New York: 2000.

46. Meins E, Fernyhough C, Russell J, Clark-Carter D. Security of attachment as a predictor of symbolic and mentalising abilities: a longitudinal study. Social Dev. 1998; 7(1):1-24.

47. Kalland M, Sinkkonen J. Finnish children in foster care: evaluating the breakdown of long-term placements. Child Welfare. 2001; 80(5):513-527. [PubMed: 11678410] 


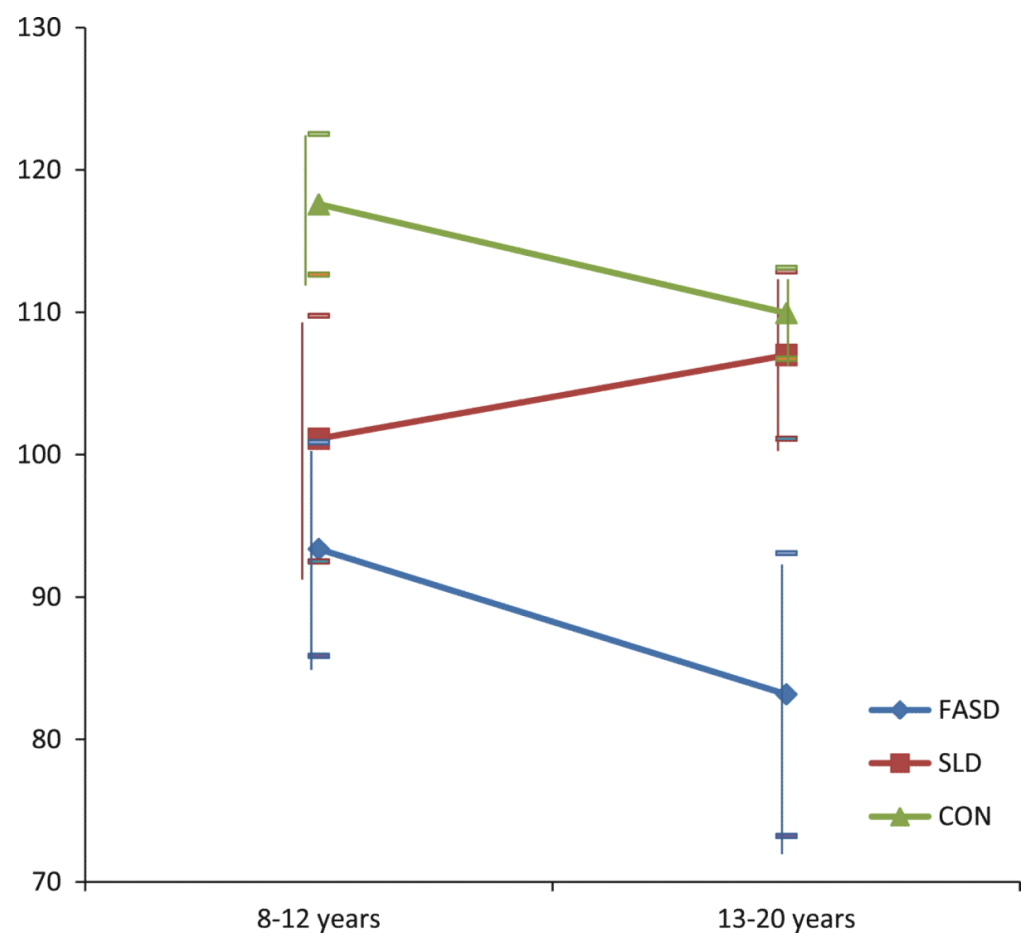

Fig. 1.

Socialization skills for FASD, SLD and CON groups in two age groups (8-12 years and 1320 years). FASD fetal alcohol spectrum disorders $(N=35$ and 37$), S L D$ specific learning disorder $(N=15$ and 15$), C O N$ typically developing controls $(N=22$ and 18$)$ 


\section{Table 1}

Demographic information and IQ data for children and adolescents with FASD, SLD, and typically developing controls $(\mathrm{CON})$

\begin{tabular}{llll}
\hline Variable & FASD & SLD & CON \\
\hline$N$ & 73 & 30 & 40 \\
Sex, $N(\%)$ & & & \\
$\quad$ Female & $44(60.3)$ & $16(53.3)$ & $22(55)$ \\
$\quad$ Male & $29(39.7)$ & $14(46.7)$ & $18(45)$ \\
Age in years M (SD) & $13.38(3.71)$ & $12.90(2.88)$ & $13.13(3.64)$ \\
WISC/WAIS IQ M (SD) & $79.34(17.27)$ & $80.23(15.86)$ & $107.32(14.49)$ \\
LIPS-R BRIEF IQ M (SD) & $90.12(16.03)$ & $87.00(14.13)$ & $108.15(12.69)$ \\
\hline
\end{tabular}

$F A S D$ fetal alcohol spectrum disorders, $S L D$ specific learning disorder, $C O N$ typically developing controls, WISC Wechsler Intelligence Scale for Children, WAIS Wechsler Intelligence Scale for Adults, LIPS-R Leiter International Performance Scale-Revised 


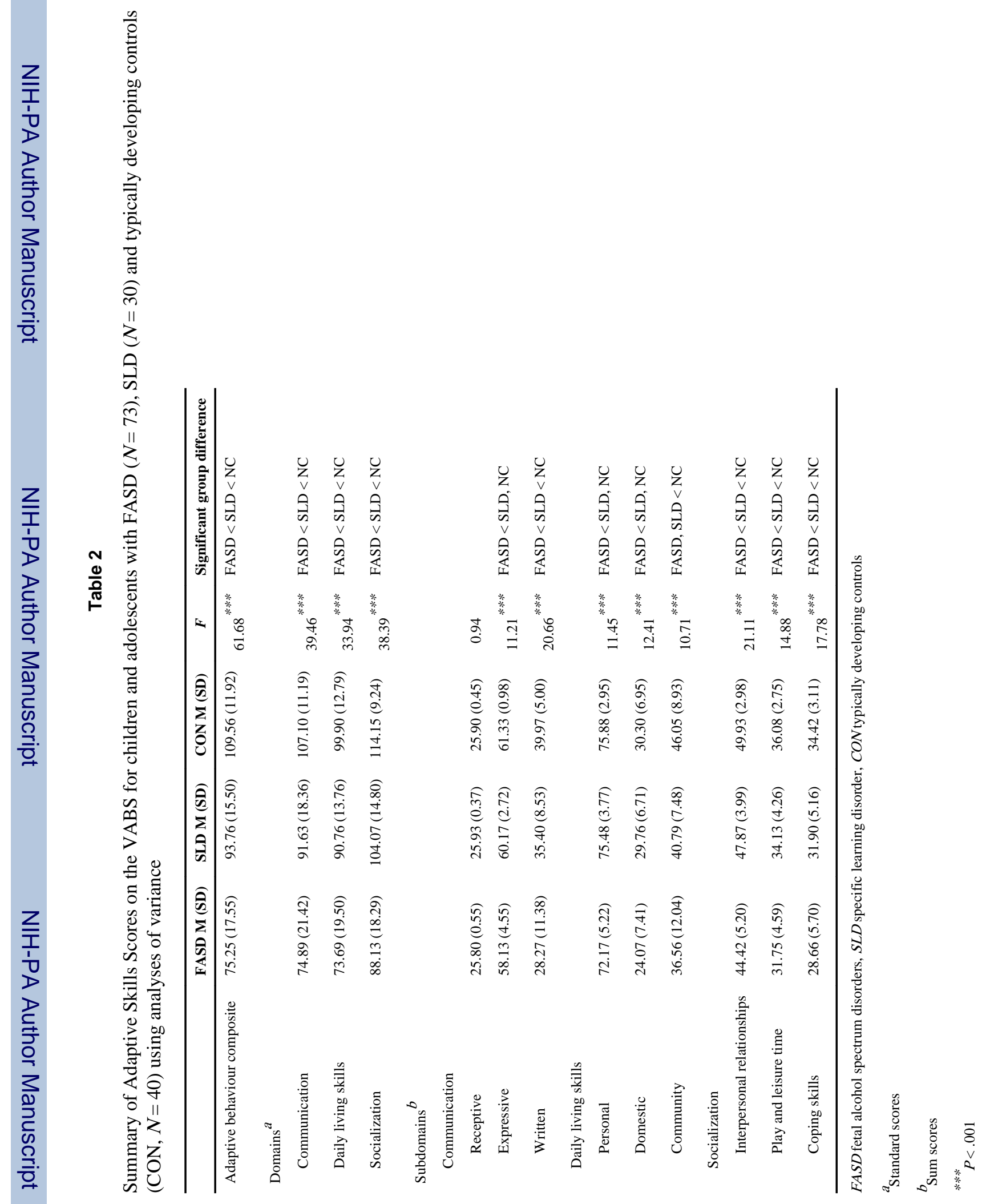




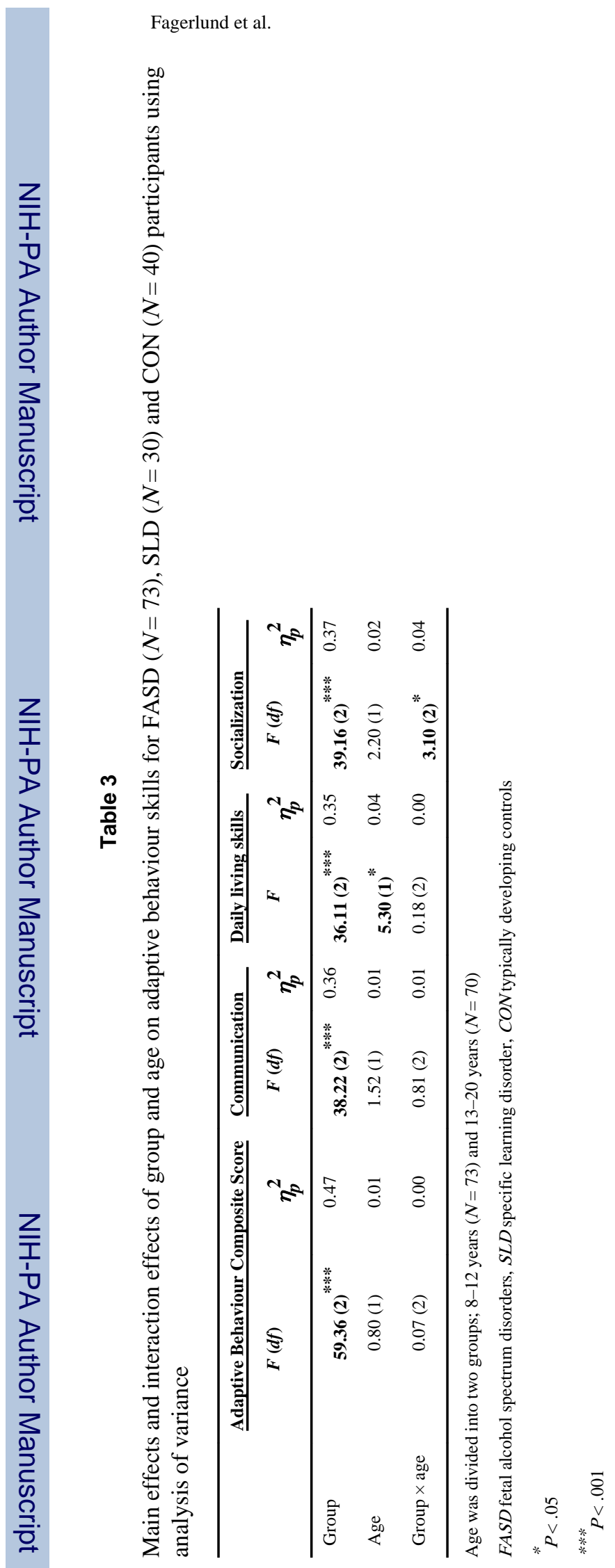

Eur Child Adolesc Psychiatry. Author manuscript; available in PMC 2013 June 17. 


\section{Table 4}

Children and adolescents with FASD and SLD living in their biological families in relation to adaptive behaviour skills as measured by the VABS

\begin{tabular}{lcc}
\hline & SLD: biological family $(\boldsymbol{N}=\mathbf{2 8})$, mean $($ SD) & FASD: biological family $(\boldsymbol{N}=\mathbf{6})$, mean $($ SD) \\
\hline Adaptive behaviour composite & $93.63(15.81)$ & $78.33(23.53)$ \\
Communication domain & $90.75(18.61)$ & $75.83(21.78)$ \\
Daily living skills domain & $91.07(13.87)$ & $73.83(29.12)$ \\
Socialization domain & $104.39(14.34)$ & $95.00(22.68)$ \\
\hline
\end{tabular}

FASD and SLD groups matched on IQ, sex and age

$F A S D$ fetal alcohol spectrum disorders, $S L D$ specific learning disorder 